\title{
Elevated Serum Levels of Soluble TNF Receptors and Adhesion Molecules Are Associated with Diabetic Retinopathy in Patients with Type-1 Diabetes
}

\author{
Shruti Sharma, ${ }^{1}$ Sharad Purohit, ${ }^{1}$ Ashok Sharma, ${ }^{1}$ Diane Hopkins, ${ }^{1}$ Leigh Steed, ${ }^{1}$ \\ Bruce Bode, ${ }^{2}$ Stephen W. Anderson, ${ }^{2}$ Ruth Caldwell, ${ }^{3}$ and Jin-Xiong She ${ }^{1}$ \\ ${ }^{1}$ Center for Biotechnology and Genomic Medicine, Georgia Regents University, 1120 15th Street, Augusta, GA 30912, USA \\ ${ }^{2}$ Pediatric Endocrine Associates, Atlanta, GA 30342, USA \\ ${ }^{3}$ Vascular Biology Center, Georgia Regents University, 1120 15th Street, Augusta, GA 30912, USA \\ Correspondence should be addressed to Shruti Sharma; shsharma@gru.edu and Jin-Xiong She; jshe@gru.edu
}

Received 7 July 2014; Revised 10 September 2014; Accepted 11 September 2014

Academic Editor: Peter Szodoray

Copyright (C) 2015 Shruti Sharma et al. This is an open access article distributed under the Creative Commons Attribution License, which permits unrestricted use, distribution, and reproduction in any medium, provided the original work is properly cited.

\begin{abstract}
Aims. To examine the association of the serum levels of TNF receptors, adhesion molecules, and inflammatory mediators with diabetic retinopathy (DR) in T1D patients. Methods. Using the multiplex immunoassay, we measured serum levels of eight proteins in 678 T1D subjects aged 20-75 years. Comparisons were made between 482 T1D patients with no complications and 196 T1D patients with DR. Results. The levels of sTNFR-I, sTNFR-II, CRP, SAA, sgp130, sIL6R, sVCAM1, and sICAM1 were significantly higher in the T1D patients with DR as compared to T1D patients with no complications. Multivariate logistic regression analysis revealed significant association for five proteins after adjustment for age, sex, and disease duration (sTNFR-I: OR = 1.57, sgp130: $\mathrm{OR}=1.43$, sVCAM1: OR $=1.27$, sICAM1: $\mathrm{OR}=1.42$, and CRP: $\mathrm{OR}=1.15)$. Conditional logistic regression on matched paired data revealed that subjects in the top quartile for sTNFR-I (OR = 2.13), sTNFR-II $(\mathrm{OR}=1.66)$, sgp130 $(\mathrm{OR}=1.82)$, sIL6R $(\mathrm{OR}=1.75)$, sVCAM1 $(\mathrm{OR}=1.98)$, sICAM1 $(\mathrm{OR}=2.23)$, CRP $(\mathrm{OR}=2.40)$ and SAA $(\mathrm{OR}=2.03)$, had the highest odds of having DR. Conclusions. The circulating markers of inflammation, endothelial injury, and TNF signaling are significantly associated with DR in patients with T1D. TNFR-I and TNFR-II receptors are highly correlated, but DR associated more strongly with TNFR-I in these patients.
\end{abstract}

\section{Introduction}

Diabetic retinopathy (DR) is a sight threatening, microvascular complication of diabetes that affects the retinal vasculature. It is the leading cause of blindness in adults 20-74 years of age in the United States with $28.5 \%$ prevalence among 40-year and older patients with diabetes [1]. According to the Centers for Disease Control and Prevention (CDC), the number of Americans aged 40 years and older with DR will triple from 5.5 million in 2005 to 16.0 million in 2050 [2]. So far the only recommended treatment for advanced retinopathy is laser photocoagulation which can control pathological neovascularization but may impair vision and in some patients the retinopathy continues to progress. Clinical trials of anti-VEGF intraocular injections have also shown promise in reducing diabetic macular edema [3]. However, these effects are usually transient and the treatment does not promote tissue repair and the need for repeated injections increases the risk of intraocular infection. Moreover, neither treatment targets early stage disease. Therefore, new markers to define the risk of type-1 diabetes (T1D) associated DR and new therapeutic targets are the critical unmet need.

Several inflammatory proteins are dysregulated in T1D [4] with inflammation being closely associated with the pathogenesis of different complications including DR [5-7]. DR is associated with several microvascular abnormalities such as leukocyte attachment to the vessel walls, occlusion of retinal capillaries, and breakdown of the blood retinal barrier and formation of acellular capillaries. The microvascular injury in DR has been linked to upregulation of several 
TABLE 1: Baseline characteristics of T1D subjects without complications and with diabetic retinopathy (DR).

\begin{tabular}{|c|c|c|c|}
\hline Patient characteristics & Without any complications & With DR & $P$ value \\
\hline Subjects $(n)$ & 482 & 196 & - \\
\hline Female (\%) & 52.1 & 61.7 & 0.027 \\
\hline Age (years) & $39.1 \pm 12.7$ & $49.3 \pm 11.6$ & $1.09 E-21$ \\
\hline Age range (years) & 20.0 to 73.8 & 24.6 to 73.8 & - \\
\hline Duration of disease & $18.2 \pm 11.1$ & $31.4 \pm 10.3$ & $1.09 E-21$ \\
\hline Systolic BP (mmHg) & $117.8 \pm 9.5$ & $122.9 \pm 12.1$ & $1.17 E-06$ \\
\hline Diastolic BP (mmHg) & $73.7 \pm 6.4$ & $73.8 \pm 7.3$ & 0.208 \\
\hline Diabetic nephropathy (\%) & 0 & 0 & - \\
\hline Diabetic neuropathy (\%) & 0 & $41.8(n=82)$ & - \\
\hline CAD (\%) & 0 & $16.3(n=32)$ & - \\
\hline Dyslipidemia (N/Y) & $376 / 106$ & $112 / 84$ & $7.05 E-08$ \\
\hline Hypertension (N/Y) & $425 / 57$ & $117 / 79$ & $1.14 E-16$ \\
\hline Hemoglobin & $14.4 \pm 1.5$ & $13.7 \pm 1.6$ & $1.40 E-04$ \\
\hline Albumin & $4.4 \pm 0.4$ & $4.2 \pm 0.4$ & $1.79 E-05$ \\
\hline $\mathrm{LDL}$ & $94.0 \pm 27.4$ & $102.4 \pm 38.3$ & 0.693 \\
\hline Total cholesterol & $174.6 \pm 34.2$ & $184.1 \pm 47.1$ & 0.565 \\
\hline Triglycerides & $90.4 \pm 67.6$ & $109.5 \pm 94.2$ & 0.395 \\
\hline $\mathrm{HDL}$ & $61.9 \pm 17.9$ & $60.7 \pm 19.4$ & 0.639 \\
\hline Creatinine & $0.9 \pm 0.2$ & $1.0 \pm 0.2$ & $7.0 E-04$ \\
\hline HbAlc & $7.8 \pm 1.1$ & $8.0 \pm 1.3$ & 0.319 \\
\hline BUN & $13.4 \pm 4.5$ & $16.4 \pm 5.6$ & $4.83 E-08$ \\
\hline Microalbumin & $24.9 \pm 122.3$ & $85.2 \pm 252.1$ & 0.017 \\
\hline
\end{tabular}

cytokines such as IL-6, IL1- $\beta$, and VEGF and pathological overexpression of adhesion molecules (ICAM-1 and VCAM-1) [8-10]. The major regulators of vascular adhesion molecules are TNF- $\alpha$ and IL-6 [11]. TNF- $\alpha$ and IL-6 are pleiotropic cytokines and key molecules in inflammatory signaling with TNF- $\alpha$ shown to be involved in the release of IL-6 [12]. IL-6 is known to induce ICAM-1 expression [13], whereas TNF- $\alpha$ leads to both ICAM-1 and VCAM-1 expression in endothelial cells [11].

Since adhesion molecules and soluble receptors of TNF- $\alpha$ and IL- 6 pathway are key mediators of endothelial activation, their elevated levels may represent risk and severity of the pathogenesis of DR. Therefore, we examined the levels of soluble TNF receptors (sTNFR-I and sTNFR-II), soluble IL6 receptors (sIL6R, sgp130), adhesion molecules (sICAM-1, sVCAM-1), and inflammatory markers (CRP, SAA) in serum of T1D patients with and without DR. The aim of this study was to examine the association of the serum levels of these inflammatory mediators with DR and to determine if these markers could be used as surrogate endpoints to define the risk of DR in T1D patients.

\section{Research Design and Methods}

2.1. Human Subjects and Serum Samples. This study was approved by the institutional review board of the Georgia Regents University, Augusta, Georgia. Blood samples from the participants of Phenome and Genome of Diabetes Autoimmunity (PAGODA) study were obtained after the informed consent from the subjects. All subjects were recruited in the state of Georgia, USA, mainly in the Atlanta and Augusta city areas. The demographic information for T1D subjects with no complications and with DR is presented in Table 1.

Peripheral blood was collected in serum separator tubes (BD Biosciences, San Jose, CA, USA) and clotted for $30 \mathrm{~min}-$ utes, the tubes were centrifuged, and serum was immediately aliquoted and stored in $-80^{\circ} \mathrm{C}$ freezers. Serum samples from T1D patients were aliquoted randomly into 96 well plates and each plate contained similar numbers of samples from T1D patients with and without DR.

2.2. Luminex Immunoassays. Luminex immunoassays for sTNFR-I, sTNFR-II, CRP, SAA, sIL6R, sgp130, sICAM-1, and sVCAM1 were obtained from Millipore (Millipore Inc., Billerica, MA, USA). Multiplex immunoassays were performed according to the manufacturer's instructions. Briefly, serum samples were incubated with antibody-coated microspheres, followed by biotinylated detection antibody. Proteins were detected by incubation with phycoerythrin-labeled streptavidin and the resultant bead immunocomplexes were read on a FLEXMAP3D (Luminex, TX, USA) with the following instrument settings: events/bead: 50 , minimum events: 0 , flow rate: $60 \mu \mathrm{L} / \mathrm{min}$, sample size: $50 \mu \mathrm{L}$, and discriminator 
gate: 8000-13500. Median fluorescence intensity (MFI) was collected and used for calculating protein concentration.

2.3. Statistical Analyses. All statistical analyses were performed using the $\mathrm{R}$ language and environment for statistical computing ( $\mathrm{R}$ version 2.15.1; R Foundation for Statistical Computing; http://www.r-project.org/). All $P$ values were two-tailed and a $P<0.05$ was considered statistically significant.

Protein concentrations were estimated using a regression fit to the standard curve with known concentration included on each plate using a serial dilution series. To achieve normal distribution, the concentrations were $\log 2$ transformed prior to all statistical analyses. The potential differences between T1D patients without any complication and T1D patients with retinopathy were initially examined using a $t$-test. The pairwise correlation between individual protein levels was computed using Pearson correlation coefficient. Clustering and visualization of correlation matrix was performed using hierarchical clustering method and heatmap. The effect of age and T1D duration on serum levels of each candidate molecule was determined using a linear regression by including age or T1D duration as covariate on data stratified by sex and disease status. To examine the relationships between retinopathy and the serum protein levels logistic regression was used. Age, sex, and T1D duration were included as covariates in a stepwise manner.

To estimate the risk of diabetes at different protein concentrations, we performed conditional logistic regression on matched paired data. Case-control matching was performed with respect to age, sex, and T1D duration using the "matching" R package [14]. The odds ratios and 95\% confidence intervals (CI) were computed for each protein and protein concentration was used as categorical variable (values 1, 2, 3, and 4 were assigned using the quartile values in controls as cutoff points).

\section{Results}

Serum levels of eight proteins in 482 T1D patients with no complications and 196 T1D patients with DR were measured. The demographic information and baseline characteristics of the subjects involved in this study are shown in Table 1 . The average age of the T1D subjects without any complications was 39.1 \pm 12.7 years and for subjects with DR was $49.3 \pm 11.6$. The duration of diabetes in patients without complications was $18.2 \pm 11.1$ as compared to $31.4 \pm 10.3$ in patients with DR.

3.1. Alterations in Serum Protein Levels in T1D Patients with $D R$. The levels of all eight molecules were significantly higher in the T1D patients with DR as compared to T1D patients with no complications: sTNFR-I (1.30-fold), sTNFR-II (1.27fold), CRP (1.53-fold), SAA (1.33-fold), sgp130 (1.14-fold), sIL6R (1.08-fold), sVCAM1 (1.11-fold), and sICAM1 (1.19fold) as shown in Figure 1. Next, we examined the pairwise correlations between all eight proteins and hierarchical clustering of the correlation matrix was performed in T1D patients with and without DR separately (Figure 2). We found three clusters of functionally related proteins with strong positive correlations. The proteins in cluster-1 include sgp130, sVCAM1, sICAM1, and sIL6R and the proteins in cluster2 are CRP and SAA. The third cluster of proteins with strong positive correlation includes sTNFR-I and sTNFR-II. The correlations were almost similar in both no complication and DR groups (Figure 2) except sTNFR-II. The correlation of sTNFR-II was increased with other proteins in DR group as compared to the T1D group without any complications.

3.2. Multivariate Logistic Regression Analysis Reveals Significant Association between Protein Levels and DR. Multivariate logistic regression analysis was performed using four different models (Model 1: no adjustments, model 2: adjusted for age, model 3: adjusted for age and sex, and model 4: adjusted for age, sex, and T1D duration). The odds ratio of five proteins showed significant association with diabetic retinopathy (Table 2 ). We found that three proteins directly involved in TNF/IL-6-pathway have larger odds ratios (sTNFR-I: OR = 1.57, sICAM1: OR = 1.42, and sgp130: $\mathrm{OR}=1.43$ ). All eight proteins have positive associations with DR for the four models (Table 2).

3.3. Risk for DR Is Directly Related to the Protein Levels. Since there was a significant effect of age, sex, and T1D duration on the protein concentrations, a paired dataset of 183 matched pairs was generated using multivariate and propensity score matching software [14]. Matching was performed with respect to age, sex, and duration of diabetes and each T1D patient with DR was paired with closest T1D patient without complication. The demographic information and baseline characteristics of the samples after matching are presented in Table 3. Conditional logistic regression was performed to estimate the risk of DR at different protein concentrations. Protein levels were used as categorical variable after dividing into 4 quartiles. The odds ratios of having DR were computed for quartile-2, quartile-3, and quartile- 4 using quartile-1 as reference. Subjects in the top quartile had the highest risk of DR compared with subjects in the bottom quartile for all eight proteins: sTNFR-I (OR = 2.13), sTNFRII $(\mathrm{OR}=1.66)$, CRP $(\mathrm{OR}=2.40)$, SAA (OR = 2.03), sgp130 $(\mathrm{OR}=1.83)$, sIL6R $(\mathrm{OR}=1.75)$, sVCAM1 $(\mathrm{OR}=1.98)$, and sICAM1 $(\mathrm{OR}=2.23)$. Also, for all proteins, an increased trend in the risk for DR was observed from quartile- 2 to quartile- 4 of protein concentrations as shown in Figure 3.

\section{Conclusions}

Hyperglycemia and aging activate multiple cellular pathways which play an important role in diabetic retinopathy. Previous studies have related inflammation and endothelial injury to be closely associated with the pathogenesis of microvascular complications including DR [5-7]. In this study, we measured serum levels of 8 proteins in blood samples from T1D patients with DR and T1D patients without any complications. We found significant alterations in the 

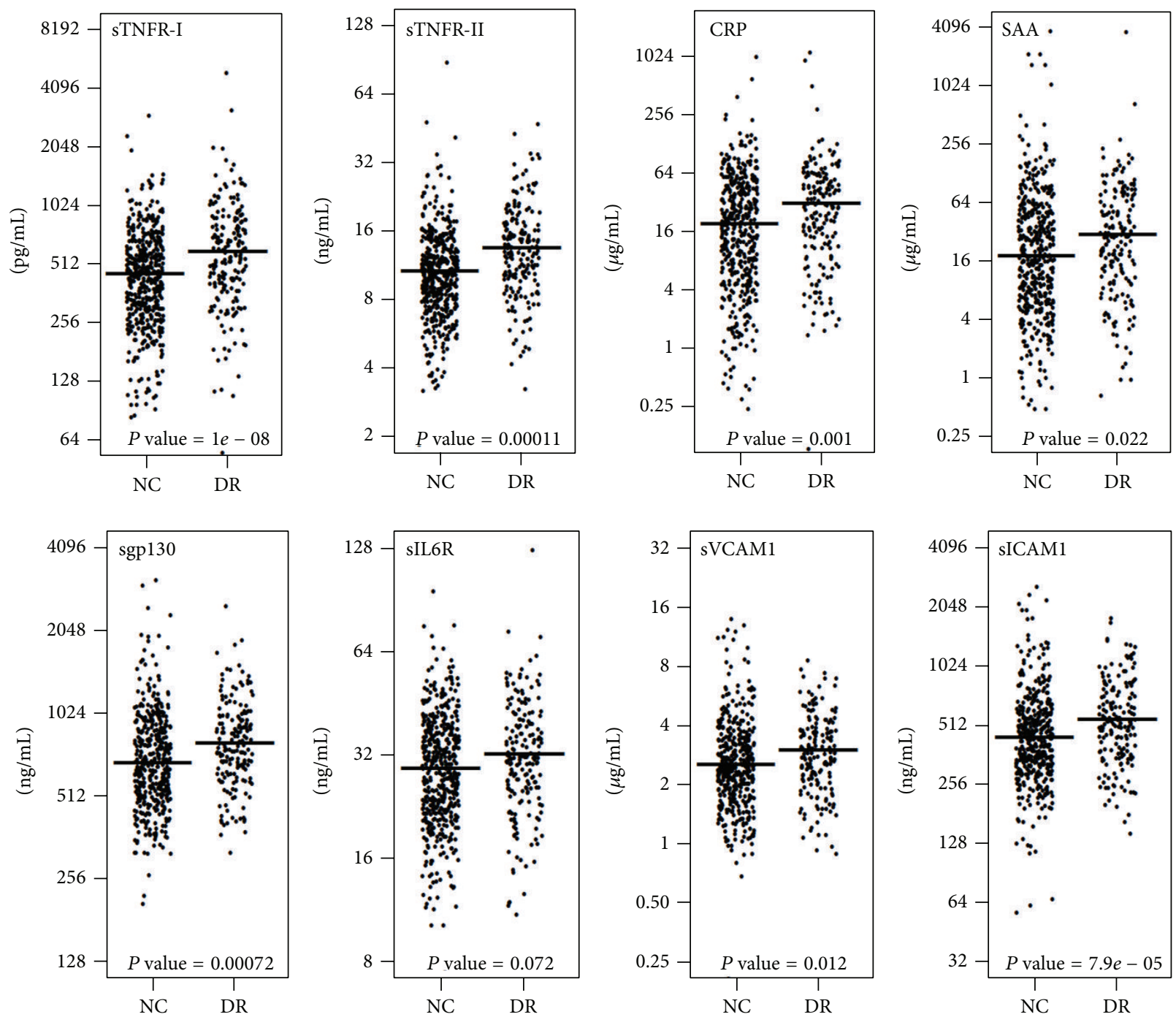

FIGURE 1: Elevated serum protein levels in T1D subjects with DR. Serum levels of eight proteins were measured in 678 T1D subjects aged 20-75 years. Comparisons were made between 482 T1D patients with no complications and 196 T1D patients with diabetic retinopathy. Plots depict the distribution of the protein levels in two different groups.

serum protein levels of sTNFR-I, sTNFR-II, CRP, SAA, sIL6R, sgp130, sVCAM1, and sICAM1.

TNF- $\alpha$ has been shown to be involved in the development and progression of DR [15]. Studies have shown the importance of TNF- $\alpha$ system in diabetic retinal microvascular damage [16]. TNF- $\alpha$ binds to its membrane receptors, TNFRI and TNFR-II, which initiate signaling pathway leading to activation of transcription factors such as NF- $\kappa$ B as well as apoptosis [17]. In animal models, drugs that target TNF$\alpha$ have been shown to reduce leukostasis, retinal vascular leakage, and retinal cell death $[18,19]$. Proteolytic cleavage of extracellular domains of TNF- $\alpha$ receptors results in their release as soluble forms (sTNFR-I and sTNFR-II). While these 2 receptors are well-known as TNF antagonist, these can also act as a reservoir of circulating TNF- $\alpha$. Recent studies have shown that these soluble forms may be more important than TNF- $\alpha$ itself in regulation of TNF signaling [20]. We found that sTNFR-I and sTNFR-II both are upregulated in
T1D patients with DR. sTNFR-I and sTNFR-II receptors were highly correlated, but DR associated more strongly with sTNFR-I in these patients.

Earlier studies have also reported that the serum and vitreous levels of sTNFRs are elevated in DR patients [21], TNFR-I expression may be a more significant target than TNF- $\alpha$ for intervention in ocular inflammation [20], and TNF- $\alpha$ inhibition is known to reduce the leukocyte adhesion in the retina and the loss of retinal microvascular cells in diabetic rats. Also, activated TNF- $\alpha$ might regulate bloodretinal barrier (BRB) breakdown, retinal leukostasis, and apoptosis in later stages of DR [22]. Thus, the effective control of TNF- $\alpha$ activity by sTNFRs within the retinal microenvironment may determine the outcome and severity of DR.

Interestingly, we found significant alterations in soluble glycoprotein 130 (sgp130) protein levels that has not been previously implicated in DR. This protein plays a crucial role 

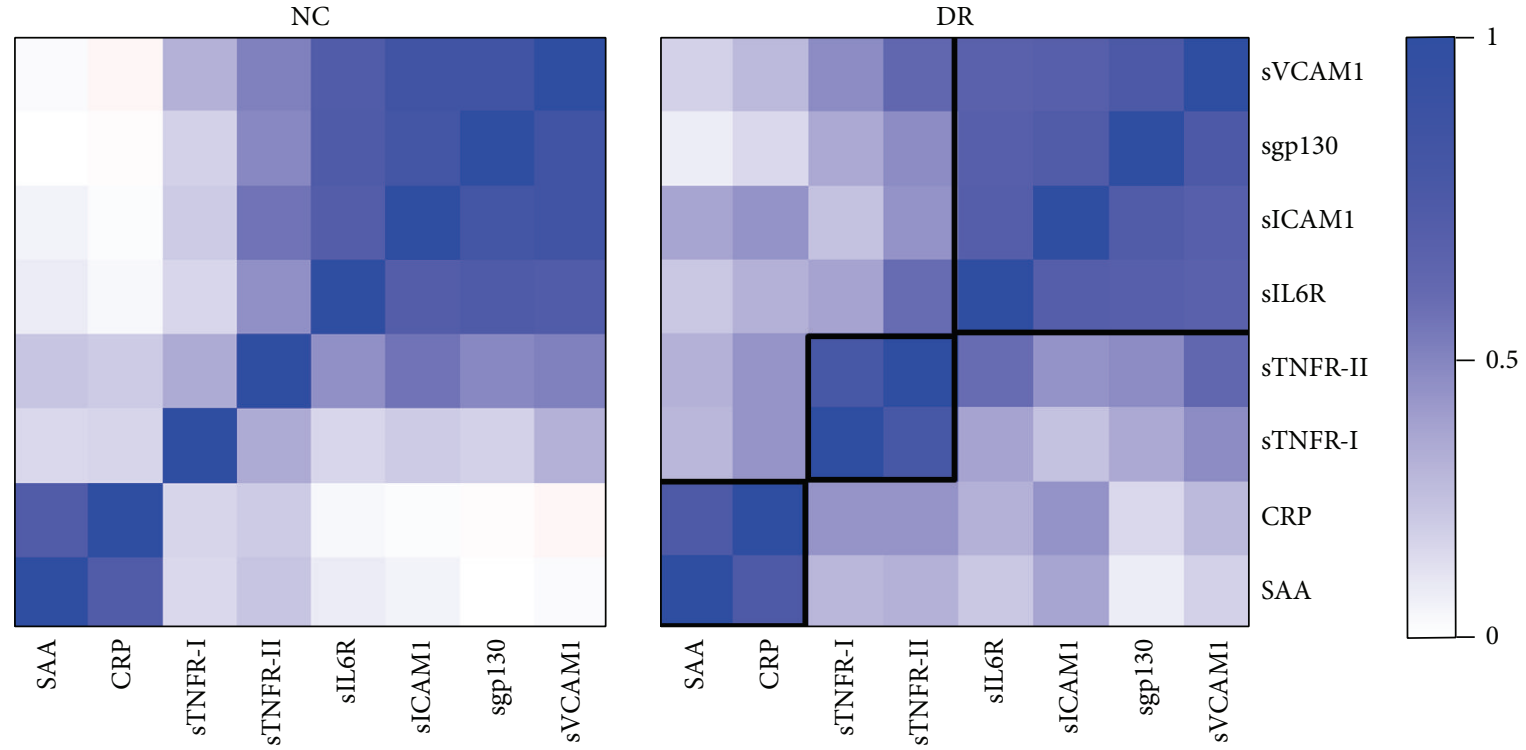

FIGURE 2: Three clusters of functionally related proteins with strong positive correlations. The pairwise correlations between all eight proteins were examined in T1D patients with and without DR separately. Correlation between individual protein levels was computed using Pearson correlation coefficient. Clustering and visualization of correlation matrix was performed using hierarchical clustering method and heatmap. Three clusters of functionally related proteins were found with strong positive correlations.

TABLE 2: Multivariate logistic regression analysis for proteins after adjustment for age, sex, and disease duration.

\begin{tabular}{|c|c|c|c|c|}
\hline & Model 1 & Model 2 & Model 3 & Model 4 \\
\hline & OR (CI 95\%) & OR (CI 95\%) & OR (CI 95\%) & OR (CI 95\%) \\
\hline & $P$ value & $P$ value & $P$ value & $P$ value \\
\hline \multirow{2}{*}{ sTNFR-I } & $1.83(1.49-2.28)$ & $1.62(1.31-2.02)$ & $1.67(1.35-2.09)$ & $1.57(1.25-2.00)$ \\
\hline & $2.67 \times 10^{-8}$ & $3.24 \times 10^{-9}$ & $4.00 \times 10^{-6}$ & $1.66 \times 10^{-4}$ \\
\hline \multirow{2}{*}{ sTNFR-II } & $1.54(1.24-1.97)$ & $1.32(1.09-1.64)$ & $1.32(1.09-1.64)$ & $1.18(0.97-1.46)$ \\
\hline & $2.46 \times 10^{-4}$ & $7.44 \times 10^{-3}$ & $7.67 \times 10^{-3}$ & 0.113 \\
\hline \multirow{2}{*}{ CRP } & $1.16(1.06-1.27)$ & $1.16(1.06-1.28)$ & $1.16(1.05-1.28)$ & $1.15(1.04-1.29)$ \\
\hline & $1.51 \times 10^{-3}$ & $2.68 \times 10^{-3}$ & $4.07 \times 10^{-3}$ & $9.37 \times 10^{-3}$ \\
\hline \multirow{2}{*}{ SAA } & $1.10(1.01-1.19)$ & $1.07(0.98-1.16)$ & $1.06(0.97-1.16)$ & $1.10(1.00-1.22)$ \\
\hline & 0.025 & 0.145 & 0.200 & 0.046 \\
\hline \multirow{2}{*}{ sgp130 } & $1.59(1.21-2.14)$ & $1.58(1.18-2.15)$ & $1.58(1.19-2.16)$ & $1.43(1.05-1.97)$ \\
\hline & $1.61 \times 10^{-3}$ & $2.71 \times 10^{-3}$ & $2.71 \times 10^{-3}$ & 0.026 \\
\hline \multirow{2}{*}{ sIL6R } & $1.26(1.00-1.63)$ & $1.27(0.99-1.69)$ & $1.28(1.00-1.70)$ & $1.25(0.96-1.66)$ \\
\hline & 0.069 & 0.077 & 0.070 & 0.108 \\
\hline \multirow{2}{*}{ sVCAM1 } & $1.30(1.05-1.64)$ & $1.28(1.01-1.63)$ & $1.28(1.01-1.63)$ & $1.27(0.98-1.65)$ \\
\hline & 0.021 & 0.044 & 0.046 & 0.074 \\
\hline \multirow{2}{*}{ sICAM1 } & $1.59(1.25-2.04)$ & $1.56(1.20-2.03)$ & $1.54(1.19-2.01)$ & $1.42(1.07-1.89)$ \\
\hline & $1.65 \times 10^{-4}$ & $8.6 \times 10^{-4}$ & $1.08 \times 10^{-3}$ & 0.015 \\
\hline
\end{tabular}

Model 1: no adjustments, model 2: adjusted for age, model 3: adjusted for age and sex, and model 4: adjusted for age, sex, and T1D duration.

in IL-6 trans-signaling. Increasing evidences suggest that IL6 pathway plays a prominent role in the pathogenesis of DR and IL- 6 and its soluble receptor (sIL-6R) operate as central regulators of the inflammatory processes $[23,24]$. The effect of IL- 6 on target cells is mediated by a complex receptor system, composed of IL-6R (gp80) and a signal-transducing glycoprotein (gp130) [25]. IL-6 signals to target cells by binding to the cell-surface IL-6R receptors known as "classic" signaling pathway. On the other hand, IL-6/sIL-6R complex can also bind to cell-surface glycoprotein 130 (gp130) on cells which do not express the IL-6R. This process has been called "IL-6 trans-signaling" mediated by gp130. The recent findings implicate IL- 6 trans-signaling in inflammation and related diseases in humans and mice [26-28]. In animal models of inflammation it has been shown that sgp130 administration decreases disease severity [29-31]. Elevated sgp130 serum 

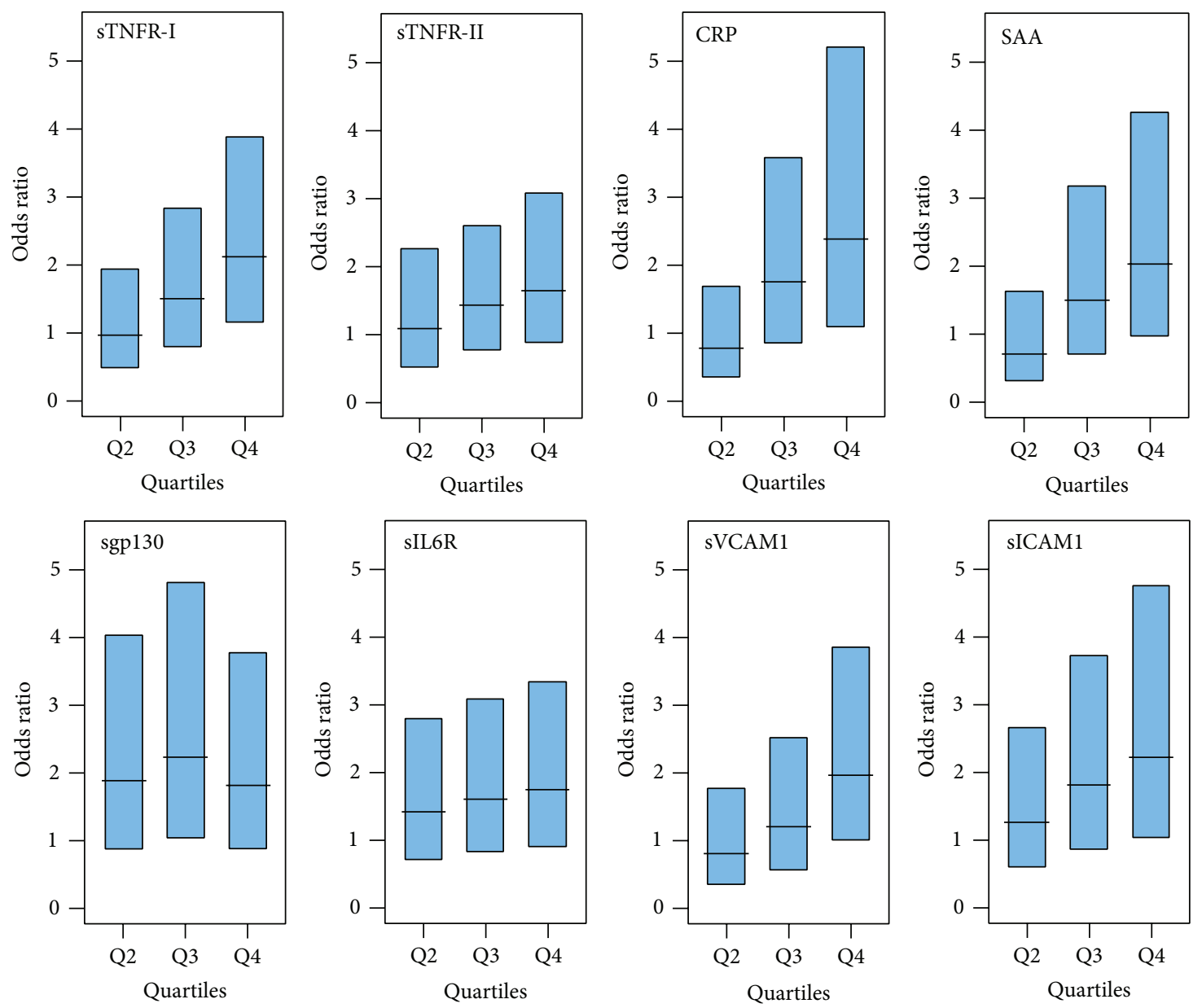

FIGURE 3: Strong association of increasing protein levels with DR. Conditional logistic regression was performed on matched paired data using cases (T1D with DR) and controls (T1D without complication) matched for age, sex, and T1D duration (183 pairs). Subjects were divided into four quartiles based on individual protein levels. The odds ratios and 95\% confidence intervals (CI) were computed for each protein using lowest quartile as referent. Compared with subjects in the bottom quartile, subjects in the top quartile had the highest risk of DR for all eight proteins. Also, an increased trend in the risk for DR was observed from quartile- 2 to quartile- 4 of protein concentrations.

TABLE 3: Baseline characteristics of T1D subjects without complications and with diabetic retinopathy after matching.

\begin{tabular}{lcc}
\hline Patient characteristics & $\begin{array}{c}\text { Without any } \\
\text { complications }\end{array}$ & $\begin{array}{c}\text { With diabetic } \\
\text { retinopathy }\end{array}$ \\
\hline Subjects ( $n)$ & 183 & 183 \\
Female (\%) & 37.7 & 37.7 \\
Age (years) & $48.15 \pm 10.7$ & $48.9 \pm 11.5$ \\
Age range (years) & 21.7 to 70.5 & 24.6 to 73.8 \\
Duration of disease & $30.7 \pm 10.7$ & $30.9 \pm 10.3$ \\
Diagnosis age & $17.8 \pm 12.4$ & $18.2 \pm 12.5$ \\
Systolic BP (mmHg) & $120.7 \pm 10.4$ & $124.1 \pm 12.2$ \\
Diastolic BP (mmHg) & $72.3 \pm 6.2$ & $72.7 \pm 8.3$ \\
Diabetic nephropathy (\%) & 0 & 0 \\
Diabetic neuropathy (\%) & 0 & $41.0(n=75)$ \\
CAD (\%) & 0 & $13.6(n=25)$ \\
\hline
\end{tabular}

concentrations were found in inflammatory diseases, such as Crohn's disease, rheumatoid arthritis, or inflammatory colon cancer [32-34]. Further studies will be required to elucidate the mechanisms of sgp130 regulation and the implications of targeting it as a therapeutic agent in DR.

The rapid, massive shedding of membrane-bound ICAM1 from EC leads to an increase in circulating soluble ICAM1 concentration which has been reported as a biomarker for inflammation and EC activation [35]. We measured these markers of endothelial activation and found them to be higher in T1D patients with DR. These molecules are expressed on the endothelial cell surface and their increased production results in recruitment and activation of granulocytes, monocytes/macrophages, and lymphocytes at the damaged tissue site [36]. These selectins also mediate initial rolling of leukocytes along the endothelium and play important roles in the firm attachment and transendothelial migration of leukocytes. TNF- $\alpha$ has been shown to induce expression of both ICAM and VCAM in endothelial cells [11]. 
Thus, our study suggests that serum sICAM1 and sVCAM1 concentrations may reflect TNF- $\alpha$ mediated progression and severity of DR associated with T1D.

Studies have also shown the association of inflammatory biomarkers such as CRP and SAA with T1D and other microvascular complications [37-40]. CRP induces proinflammatory effects through overproduction of ICAM-1 and VCAM-1 adhesion molecules. Also, IL-6 activation leads to production of CRP which has been previously shown to be elevated in adults with T1D [37, 41]. In the Diabetes Autoimmunity Study of the Young (DAISY), elevated CRP levels were more frequent in children who later developed T1D and provide evidence that the disease is an immunoinflammatory disorder [37]. CRP is shown to mediate endothelial dysfunction by inhibiting endothelium-dependent NOmediated dilation in retinal arterioles by producing superoxide from NADPH oxidase [42]. Recently, a study in a rat model has shown that elevated CRP levels are associated with increased cardiovascular events and endothelial dysfunction [43]. Another study has reported that elevated CRP levels in T1D patients were not associated with glycemic control but reflected a low-grade inflammation associated with the activation of innate immune activity [37]. In our study, circulating levels of CRP and SAA (a similar inflammatory marker) were increased in T1D subjects with DR as compared to T1D patients with no complications.

In conclusion, this study reveals that serum levels of TNF receptors, adhesion molecules, and other inflammatory mediators could be used as surrogate endpoints in studies of interventions to decrease inflammation among subjects with T1D. Significant associations between systemic markers of inflammation highlight that subclinical inflammation might be a mechanism through which hyperglycemia causes DR with endothelial impairment playing an important role in the pathogenesis of DR. However, future studies will be required to determine the precise understanding of whether these elevated biomarkers are participating in or are an indicator of DR progression.

\section{Conflict of Interests}

The authors declare that there is no conflict of interests regarding the publication of this paper.

\section{Acknowledgments}

This work was supported by grants from the National Institutes of Health (4R33HD050196, 4R33-DK069878, and 2RO1HD37800) and Juvenile Diabetes Research Foundation (JDRF 1-2004-661) to Dr. Jin-Xiong She. Sharad Purohit is a recipient of a JDRF Advanced Postdoctoral Fellowship (JDRF 10-2006-792) and Career Development Award (JDRF 2-2011153).

\section{References}

[1] X. Zhang, J. B. Saaddine, C.-F. Chou et al., "Prevalence of diabetic retinopathy in the United States, 2005-2008," Journal of the American Medical Association, vol. 304, no. 6, pp. 649656, 2010.

[2] J. B. Saaddine, A. A. Honeycutt, K. M. V. Narayan, X. Zhang, R. Klein, and J. P. Boyle, "Projection of diabetic retinopathy and other major eye diseases among people with diabetes mellitus: United States, 2005-2050," Archives of Ophthalmology, vol. 126, no. 12, pp. 1740-1747, 2008.

[3] B. P. Nicholson and A. P. Schachat, "A review of clinical trials of anti-VEGF agents for diabetic retinopathy," Graefe's Archive for Clinical and Experimental Ophthalmology, vol. 248, no. 7, pp. 915-930, 2010.

[4] B. Baumann, H. H. Salem, and B. O. Boehm, "Antiinflammatory therapy in type 1 diabetes," Current Diabetes Reports, vol. 12, no. 5, pp. 499-509, 2012.

[5] D. Gologorsky, A. Thanos, and D. Vavvas, "Therapeutic interventions against inflammatory and angiogenic mediators in proliferative diabetic retinopathy," Mediators of Inflammation, vol. 2012, Article ID 629452, 10 pages, 2012.

[6] D. Bending, P. Zaccone, and A. Cooke, "Inflammation and type one diabetes," International Immunology, vol. 24, no. 6, pp. 339346, 2012.

[7] A. P. Adamis, "Is diabetic retinopathy an inflammatory disease?" British Journal of Ophthalmology, vol. 86, no. 4, pp. 363$365,2002$.

[8] C. Gustavsson, C.-D. Agardh, A. V. Zetterqvist, J. Nilsson, E. Agardh, and M. F. Gomez, "Vascular cellular adhesion molecule-1 (VCAM-1) expression in mice retinal vessels is affected by both hyperglycemia and hyperlipidemia," PLoS ONE, vol. 5, no. 9, Article ID e12699, pp. 1-12, 2010.

[9] V. Videm and M. Albrigtsen, "Soluble ICAM-1 and VCAM-1 as markers of endothelial activation," Scandinavian Journal of Immunology, vol. 67, no. 5, pp. 523-531, 2008.

[10] S. S. Soedamah-Muthu, N. Chaturvedi, C. G. Schalkwijk, C. D. A. Stehouwer, P. Ebeling, and J. H. Fuller, "Soluble vascular cell adhesion molecule-1 and soluble E-selectin are associated with micro- and macrovascular complications in Type 1 diabetic patients," Journal of Diabetes and its Complications, vol. 20, no. 3, pp. 188-195, 2006.

[11] M. P. Bevilacqua, "Endothelial-leukocyte adhesion molecules," Annual Review of Immunology, vol. 11, pp. 767-804, 1993.

[12] S. B. Ng, Y. H. Tan, and G. R. Guy, "Differential induction of the interleukin-6 gene by tumor necrosis factor and interleukin-1," Journal of Biological Chemistry, vol. 269, no. 29, pp. 19021-19027, 1994.

[13] M. Romano, M. Sironi, C. Toniatti et al., "Role of IL-6 and its soluble receptor in induction of chemokines and leukocyte recruitment," Immunity, vol. 6, no. 3, pp. 315-325, 1997.

[14] J. S. Sekhon, "Multivariate and propensity score matching software with automated balance optimization: the matching package for R," Journal of Statistical Software, vol. 42, no. 7, pp. $1-52,2011$.

[15] X.-L. Chen, X.-D. Zhang, Y.-Y. Li, X.-M. Chen, D.-R. Tang, and R.-J. Ran, "Involvement of HMGB1 mediated signalling pathway in diabetic retinopathy: Evidence from type 2 diabetic rats and ARPE-19 cells under diabetic condition," British Journal of Ophthalmology, vol. 97, no. 12, pp. 1598-1603, 2013.

[16] Y. Behl, P. Krothapalli, T. Desta, A. DiPiazza, S. Roy, and D. T. Graves, "Diabetes-enhanced tumor necrosis factor- $\alpha$ production promotes apoptosis and the loss of retinal microvascular cells in type 1 and type 2 models of diabetic retinopathy," The American Journal of Pathology, vol. 172, no. 5, pp. 1411-1418, 2008. 
[17] D. Aderka, "The potential biological and clinical significance of the soluble tumor necrosis factor receptors," Cytokine and Growth Factor Reviews, vol. 7, no. 3, pp. 231-240, 1996.

[18] A. M. Joussen, V. Poulaki, N. Mitsiades et al., "Nonsteroidal anti-inflammatory drugs prevent early diabetic retinopathy via TNF-alpha suppression," The FASEB Journal, vol. 16, no. 3, pp. 438-440, 2002.

[19] A. M. Joussen, S. Doehmen, M. L. Le et al., "TNF- $\alpha$ mediated apoptosis plays an important role in the development of early diabetic retinopathy and long-term histopathological alterations," Molecular Vision, vol. 15, pp. 1418-1428, 2009.

[20] E. B. Cook, J. L. Stahl, F. M. Graziano, and N. P. Barney, "Regulation of the receptor for TNF $\alpha$, TNFR1, in human conjunctival epithelial cells," Investigative Ophthalmology and Visual Science, vol. 49, no. 9, pp. 3992-3998, 2008.

[21] G. A. Limb, R. D. Hollifield, L. Webster, D. G. Charteris, and A. H. Chignell, "Soluble TNF receptors in vitreoretinal proliferative disease," Investigative Ophthalmology and Visual Science, vol. 42, no. 7, pp. 1586-1591, 2001.

[22] H. Huang, J. K. Gandhi, X. Zhong et al., "TNF $\alpha$ is required for late BRB breakdown in diabetic retinopathy, and its inhibition prevents leukostasis and protects vessels and neurons from apoptosis," Investigative Ophthalmology and Visual Science, vol. 52, no. 3, pp. 1336-1344, 2011.

[23] C. Gabay, "Interleukin-6 and chronic inflammation," Arthritis Research and Therapy, vol. 8, article S3, supplement 2, 2006.

[24] M. Rincon, "Interleukin-6: From an inflammatory marker to a target for inflammatory diseases," Trends in Immunology, vol. 33, no. 11, pp. 571-577, 2012.

[25] T. Taga, M. Hibi, Y. Hirata et al., "Interleukin-6 triggers the association of its receptor with a possible signal transducer, gp130," Cell, vol. 58, no. 3, pp. 573-581, 1989.

[26] N. Ebihara, A. Matsuda, S. Nakamura, H. Matsuda, and A. Murakami, "Role of the IL-6 classic-and trans-signaling pathways in corneal sterile inflammation and wound healing," Investigative Ophthalmology and Visual Science, vol. 52, no. 12, pp. 8549-8557, 2011.

[27] L.-H. Wei, C.-H. Chou, M.-W. Chen et al., "The role of IL6 trans-signaling in vascular leakage: implications for ovarian hyperstimulation syndrome in a murine model," Journal of Clinical Endocrinology and Metabolism, vol. 98, no. 3, pp. E472E484, 2013.

[28] D. T. Fisher, Q. Chen, J. J. Skitzki et al., "IL-6 trans-signaling licenses mouse and human tumor microvascular gateways for trafficking of cytotoxic T cells," Journal of Clinical Investigation, vol. 121, no. 10, pp. 3846-3859, 2011.

[29] P. J. Richards, M. A. Nowell, S. Horiuchi et al., "Functional characterization of a soluble gp130 isoform and its therapeutic capacity in an experimental model of inflammatory arthritis," Arthritis and Rheumatism, vol. 54, no. 5, pp. 1662-1672, 2006.

[30] K. Mitsuyama, S. Matsumoto, S. Rose-John et al., "STAT3 activation via interleukin 6 trans-signalling contributes to ileitis in SAMP1/Yit mice," Gut, vol. 55, no. 9, pp. 1263-1269, 2006.

[31] S. Thiem, T. P. Pierce, M. Palmieri et al., "mTORC1 inhibition restricts infammation-associated gastrointestinal tumorigenesis in mice," The Journal of Clinical Investigation, vol. 123, no. 2, pp. 767-781, 2013.

[32] R. Atreya, J. Mudter, S. Finotto et al., "Blockade of interleukin 6 trans signaling suppresses T-cell resistance against apoptosis in chronic intestinal inflammation: evidence in crohn disease and experimental colitis in vivo," Nature Medicine, vol. 6, no. 5, pp. 583-588, 2000.
[33] T. Robak, A. Gladalska, H. Stepień, and E. Robak, "Serum levels of interleukin-6 type cytokines and soluble interleukin6 receptor in patients with rheumatoid arthritis," Mediators of Inflammation, vol. 7, no. 5, pp. 347-353, 1998.

[34] S. Rose-John, K. Mitsuyama, S. Matsumoto, W. M. Thaiss, and J. Scheller, "Interleukin-6 trans-signaling and colonic cancer associated with inflammatory bowel disease," Current Pharmaceutical Design, vol. 15, no. 18, pp. 2095-2103, 2009.

[35] G. Liu, A. T. Place, Z. Chen et al., "ICAM-1-activated Src and eNOS signaling increase endothelial cell surface PECAM-1 adhesivity and neutrophil transmigration," Blood, vol. 120, no. 9, pp. 1942-1952, 2012.

[36] J. F. McHale, O. A. Harari, D. Marshall, and D. O. Haskard, "Vascular endothelial cell expression of ICAM-1 and VCAM1 at the onset of eliciting contact hypersensitivity in mice: evidence for a dominant role of TNF- $\alpha$, Journal of Immunology, vol. 162, no. 3, pp. 1648-1655, 1999.

[37] H. P. Chase, S. Cooper, I. Osberg et al., "Elevated C-reactive protein levels in the development of type 1 diabetes," Diabetes, vol. 53, no. 10, pp. 2569-2573, 2004.

[38] P. Fasching, M. Veitl, M. Rohac et al., "Elevated concentrations of circulating adhesion molecules and their association with microvascular complications in insulin-dependent diabetes mellitus," Journal of Clinical Endocrinology and Metabolism, vol. 81, no. 12, pp. 4313-4317, 1996.

[39] R. H. Muni, R. P. Kohly, E. Q. Lee, J. E. Manson, R. D. Semba, and D. A. Schaumberg, "Prospective study of inflammatory biomarkers and risk of diabetic retinopathy in the diabetes control and complications trial," JAMA Ophthalmology, vol. 131, no. 4, pp. 514-521, 2013.

[40] A. J. Overgaard, J. N. McGuire, P. Hovind, H.-H. Parving, P. Rossing, and F. Pociot, "Serum amyloid A and C-reactive protein levels may predict microalbuminuria and macroalbuminuria in newly diagnosed type 1 diabetic patients," Journal of Diabetes and Its Complications, vol. 27, no. 1, pp. 59-63, 2013.

[41] E. S. Kilpatrick, B. G. Keevil, C. Jagger, R. J. Spooner, and M. Small, "Determinants of raised C-reactive protein concentration in type 1 diabetes," QJM-Monthly Journal of the Association of Physicians, vol. 93, no. 4, pp. 231-236, 2000.

[42] T. Nagaoka, L. Kuo, Y. Ren, A. Yoshida, and T. W. Hein, "Creactive protein inhibits endothelium-dependent nitric oxidemediated dilation of retinal arterioles via enhanced superoxide production," Investigative Ophthalmology and Visual Science, vol. 49, no. 5, pp. 2053-2060, 2008.

[43] T. W. Hein, U. Singh, J. Vasquez-Vivar, S. Devaraj, L. Kuo, and I. Jialal, "Human C-reactive protein induces endothelial dysfunction and uncoupling of eNOS in vivo," Atherosclerosis, vol. 206, no. 1, pp. 61-68, 2009. 


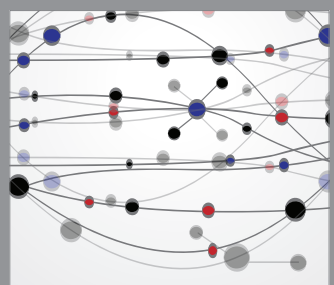

The Scientific World Journal
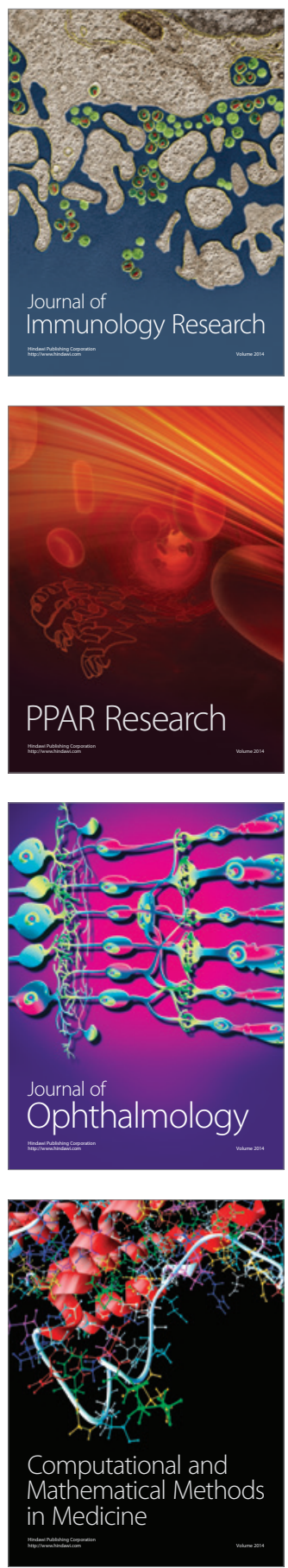

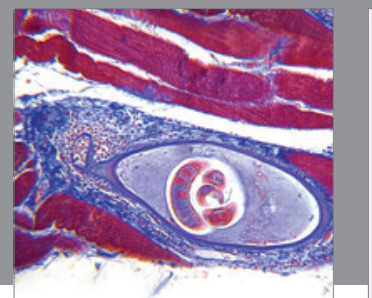

Gastroenterology

Research and Practice
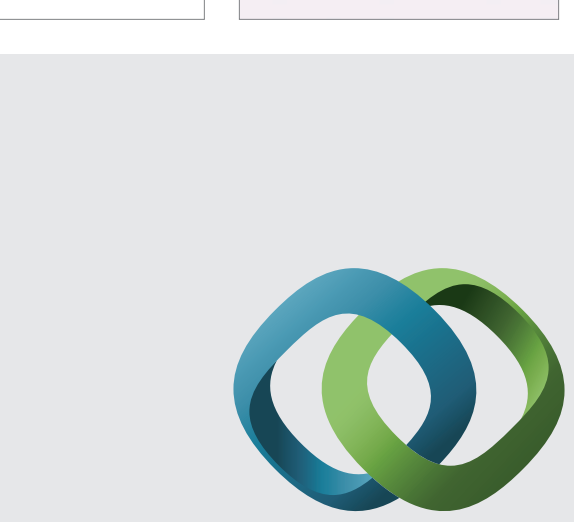

\section{Hindawi}

Submit your manuscripts at

http://www.hindawi.com
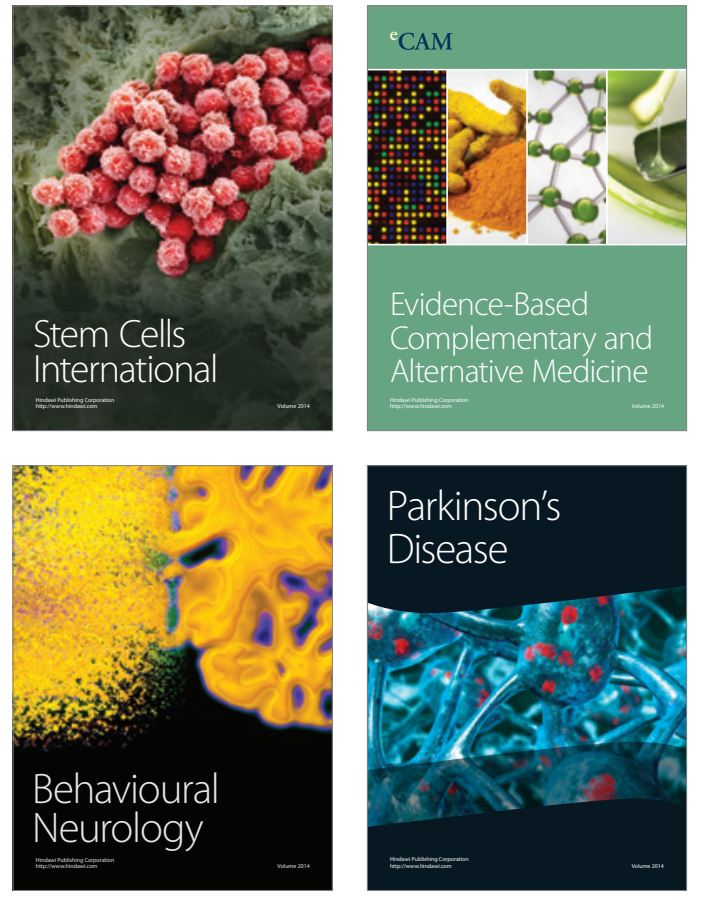
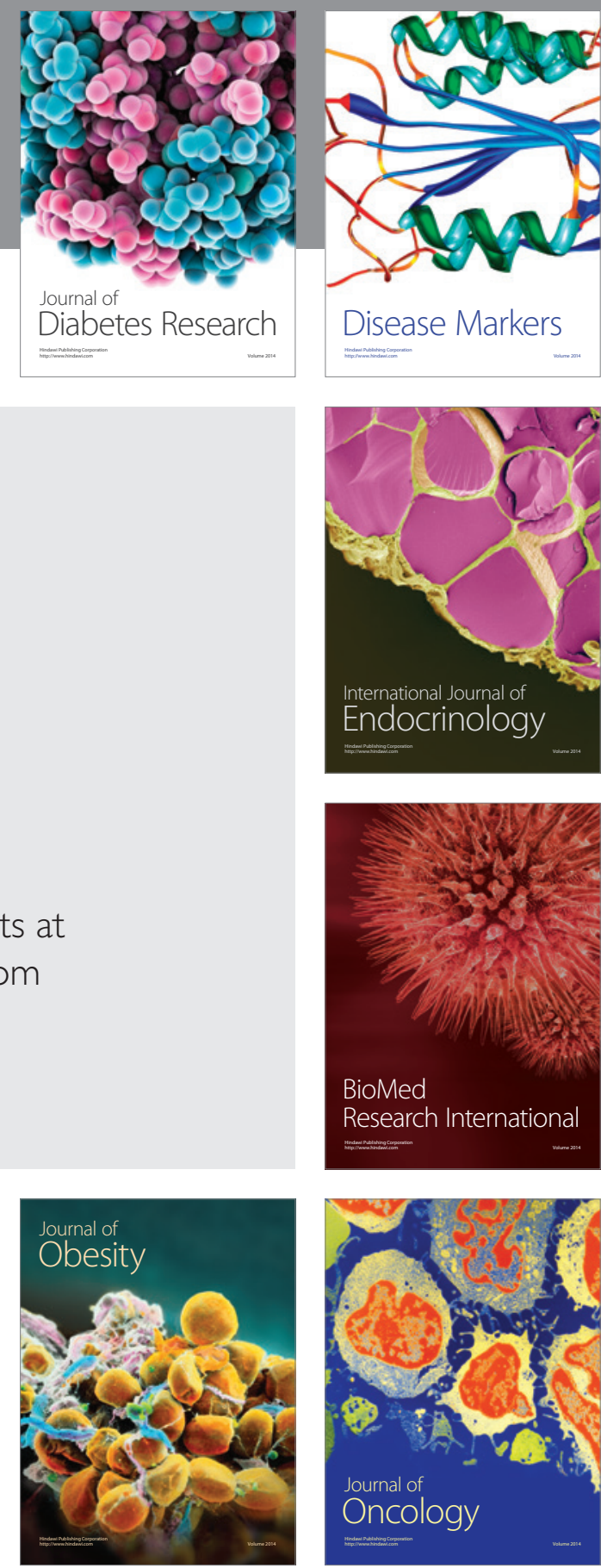

Disease Markers
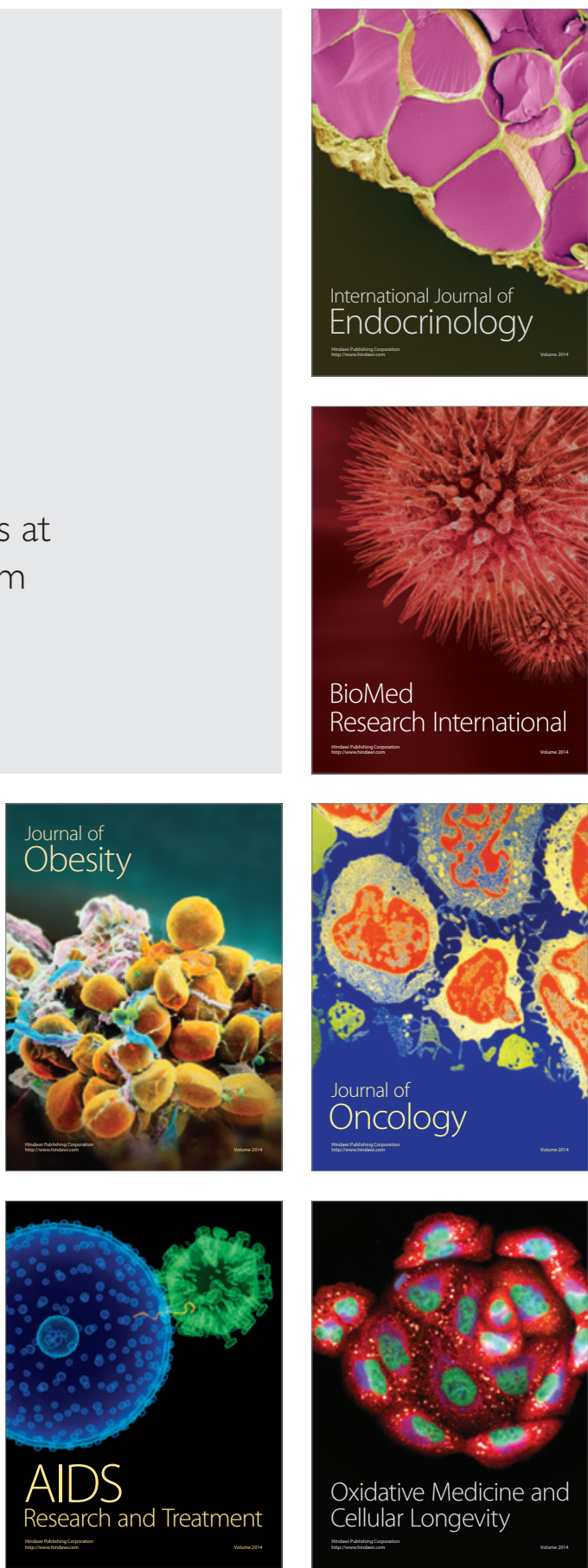